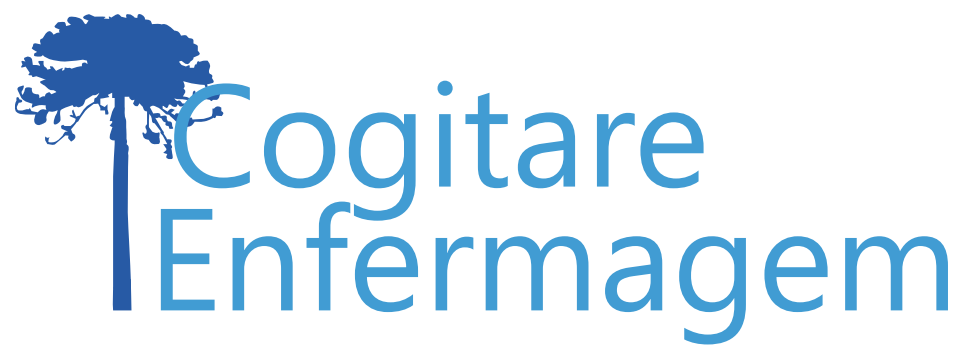

\title{
O PROCESSO DE MORRER E MORTE DE PACIENTES COM COVID-19: UMA REFLEXÃO À LUZ DA ESPIRITUALIDADE
}

Maria da Conceição Quirino dos Santos da Silva ${ }^{1}$ Alba Benemérita Alves Vilela², Rita Narriman Silva de Oliveira Boery ${ }^{3}$, Rudval Souza da Silva ${ }^{4}$

\section{RESUMO}

Objetivo: refletir sobre o processo de morrer e morte de pacientes com Covid-19 à luz da espiritualidade.

Desenvolvimento: em meio à pandemia provocada pelo novo Coronavírus, mais uma vez nos defrontamos com a temida morte, com um agravante: mortes em números crescentes e marcadas pela ausência da oportunidade de um momento para despedidas, pacientes morrendo em companhia apenas dos profissionais de saúde, e os corpos seguindo direto para os cemitérios. Cabenos discutir um elemento pelo qual a maioria das pessoas busca uma fortaleza, a espiritualidade. Considerações finais: importa compreender o sentido da espiritualidade por parte dos profissionais de saúde, em busca do apoio aos pacientes em processo de morrer e às famílias enlutadas, o que a torna um potencial integrador e harmonizador das relações interpessoais.

DESCRITORES: Covid-19; Pandemias; Cuidados paliativos na terminalidade da vida; Espiritualidade; Enfermagem.

Silva M da CQ dos S da, Vilela ABA, Silva RS da, Boery RNS de O. O processo morrer e morte de pacientes com Covid-19: uma reflexão à luz da espiritualidade. Cogitare enferm. [Internet]. 2020 [acesso em "colocar data de acesso, dia, mês abreviado e ano"]; 25. Disponível em: http://dx.doi.org/10.5380/ce.v25i0.73571.

${ }^{1}$ Enfermeira. Doutoranda em Enfermagem. Universidade Estadual do Sudoeste da Bahia. Jequié, BA, Brasil. ${ }^{2}$ Enfermeira. Doutora em Enfermagem. Docente da Universidade Estadual do Sudoeste da Bahia. Jequié, BA, Brasil. 9 ${ }^{3}$ Enfermeira. Doutora em Enfermagem. Pós-Doutoramento em Bioética. Docente da Universidade Estadual do Sudoeste da Bahia. Jequié, BA, Brasil. 9

${ }^{4}$ Enfermeiro. Doutor em Enfermagem. Docente da Universidade do Estado da Bahia/Campus VII. Senhor do Bonfim, BA, Brasil. () 


\title{
THE DYING PROCESS AND DEATH OF PATIENTS WITH COVID-19: A REFLECTION IN THE LIGHT OF SPIRITUALITY
}

\author{
ABSTRACT \\ Objective: to reflect on the dying process and death of patients with Covid-19 in the light of \\ spirituality. \\ Development: Amid the pandemic caused by the new Coronavirus, we once again face the \\ feared death, with an aggravating factor: increasing number of deaths characterized by the \\ lack of opportunity for the individuals to say goodbye to their loved ones, dying patients \\ accompanied only by health professionals, and bodies taken directly to the cemeteries. It is \\ worth discussing here an element through which most people seek strength: spirituality. \\ Final considerations: Understanding the sense of spirituality on the part of health professionals, \\ in search of support for dying patients and bereaved families is important, as spirituality can \\ be a potential factor of integration and harmonization of interpersonal relationships.
}

DESCRIPTORS: Covid-19; Pandemics; Palliative care at the end of life; Spirituality; Nursing.

\section{EL PROCESO DE MURIR Y DE MUERTE DE PACIENTES CON COVID-19: UNA REFLEXIÓN A LA LUZ DE LA ESPIRITUALIDAD}

\begin{abstract}
RESUMEN:
Objetivo: reflexionar acerca del proceso de morir y de muerte de pacientes con Covid-19 a la luz de la espiritualidad.

Desarrollo: en razón de la pandemia provocada por el nuevo Coronavirus, una vez más uno se queda delante de la muerte, pero con un agravante: muertes en números crecientes y señaladas por la ausencia da oportunidad de un momento para despedidas, pacientes muriendo solos, en compañía únicamente de los profesionales de salud, y los cuerpos siguiendo directamente a los cementerios. El objetivo es, por lo tanto, discutir un elemento por el cual la mayoría de las personas busca una fuente de fuerza, la espiritualidad.

Consideraciones finales: es importante comprender el sentido de la espiritualidad por parte de los profesionales de salud, en búsqueda del apoyo a los pacientes en proceso de morir y a las familias enlutadas, lo que los vuelve un potencial integrador y armonizador de las relaciones interpersonales.
\end{abstract}

DESCRIPTORES: Covid-19; Pandemias; Cuidados paliativos en la finitud de la vida; Espiritualidad; Enfermería. 
A saúde mundial enfrenta um problema avassalador iniciado em novembro de 2019 na cidade de Wuhan, na China, caracterizado por contaminação respiratória grave, o qual somente em janeiro de 2020 foi identificado pelas autoridades sanitárias chinesas como o novo Coronavírus. Rapidamente, em março do corrente ano (2020), a Organização Mundial de Saúde (OMS) anuncia a presença de uma pandemia - emergência de saúde pública de interesse internacional, havendo na ocasião da declaração mais de 80.000 casos espalhados por todo mundo, não restando países sem casos confirmados ${ }^{(1)}$.

Em decorrência da pandemia, dois meses após a identificação do vírus na China, o sistema de saúde italiano entra em colapso. Naquele momento, a Itália era o país onde se concentrava o segundo maior número de idosos no mundo, sendo o Japão o primeiro estatisticamente $^{(2)}$.

A Covid-19, um tipo gravíssimo de pneumonia, tem os idosos como população alvo, seguido e/ou associado a pessoas com doenças pré-existentes como cardiopatias, diabetes e hipertensão. O Brasil enfrenta uma situação preocupante: pesquisas divulgadas em fevereiro de 2020 já expunham a possibilidade do país de entrar em colapso em final de abril a início de maio, caso medidas de isolamento e/ou distanciamento social não fossem adotadas em tempo oportuno ${ }^{(3)}$.

Assim, instala-se uma das maiores pandemias de nossos tempos, a Covid-19, ainda em expansão no planeta e crescendo assustadoramente. Enquanto essa reflexão estava sendo redigida, inúmeros casos surgiam a cada dia, elevando os números de mortos. A Itália, assim como o Brasil, foi o país atingido em demasia. Na cidade italiana de Emiliana de Piacenza, deu-se a busca pelo paciente zero, a identidade do desconhecido vírus ${ }^{(4)}$. Enquanto isso, os hospitais da Inglaterra foram instruídos pelo governo a suspender todas as cirurgias eletivas não urgentes por pelo menos três meses, como medida para ajudar o serviço a lidar com a pandemia ${ }^{(5)}$.

Certamente, a temeridade do processo de morrer pela Covid-19 se alarga com a idade, entendendo que o maior número de mortes é de idosos, notadamente em pessoas portadoras de doenças crônicas ${ }^{(6-7)}$. A redução do campo imunológico populacional senescente amplia a vulnerabilidade às doenças infectocontagiosas e, consequentemente, os prognósticos antagônicos para aqueles portadores de enfermidades crônicas ${ }^{(6,8)}$. Nessa convivência, ações globais, nas quais o Brasil estava incluído, de medidas de isolamento e/ ou distanciamento social foram adotadas, com interrupção de aulas e afazeres presenciais não essenciais.

Tais medidas comprometeram grande parcela da população e ocasionaram danos emocionais e financeiros, que se agravaram quando somados ao sofrimento com a dor emocional da perda e maior número de hospitalizações ${ }^{(6)}$.

Os fatos em cada país surgiram em momentos diferenciados, contudo, seguindo o mesmo curso pandêmico. O processo de morrer e a temeridade da morte, por parte dos doentes, familiares e profissionais de saúde, passa a ser foco dos noticiários, e não se fala mais na morte de uma pessoa, mas em números de mortes. A morte se distancia do seio familiar, alcançando um espaço tenebroso das estatísticas. Aqui, vale destacar, que a mesma morte se diferencia quando ela chega à família de cada um de nós, a dor emocional associada à comoção social se multiplica e passa a ser uma dor familiar. É esse momento que nos remete à discussão da dor espiritual, uma relação transcendental da alma com a divindade, e à mudança decorrente do cenário pandêmico. Nessa perspectiva, o presente estudo tem por objetivo refletir sobre o processo de morrer e morte de pacientes com Covid-19 à luz da espiritualidade. 
O planeta atravessa uma fase complexa e longe de obter respostas concretas para conter o surto da pandemia pelo novo Coronavírus. A Covid-19, doença causada pelo SARS-CoV-2 (Severe Acute Respiratory Syndrome Coronavírus 2), apresenta período médio de incubação de 5,5 dias e aparecimento de sintomas em torno de 11 a 14 dias $^{(9-10)}$.

O avanço de casos no mundo tem sido acompanhado e registrado pela OMS, minuto a minuto, juntamente com a formulação de planos para respostas rápidas à disseminação da doença(11-12). Até o dia 11 de maio de 2020, foram mais de 3.917.366 casos confirmados, com 274.361 mortes registradas no mundo; desse total, o Brasil contava à época com 162.699 casos e 11.123 mortes $^{(11)}$.

O número elevado de mortes vem preocupando as autoridades governamentais de todo o mundo. Devido ao alto grau de transmissibilidade, as vítimas em estado grave são afastadas dos seus familiares, sem direito a visitas. Nesse ínterim, o processo de morrer e a morte ocorrem muito rápido e, pior, sem direito a despedidas ${ }^{(13)}$.

A morte é um fenômeno que suscita angústia, medo e exacerbação da ansiedade ${ }^{(14)}$. Embora seja parte da vida, ela ainda é um tabu, haja visto não sermos educados acerca do como melhor lidar com a morte ${ }^{(14-15)}$. As crenças sobre a morte sofrem influências do meio social, cultural e de pensamentos filosóficos que, conscientemente ou não, moldam nossas condutas. Em situações difíceis, os profissionais da saúde se deparam com o sofrimento físico, emocional, social e espiritual diante da morte do outro, o que remete ao reflexo da sua própria morte ${ }^{(16)}$.

Pensando no cuidar como um conceito epistêmico multidimensional, entende-se que é necessário refletir acerca da dimensão espiritual, reconhecendo sua importância em como lidar com o incerto, com agravamento de doenças como a Covid-19 e a finitude da vida $^{(17-18)}$. O bem-estar espiritual visa a oferecer amparo diante do desespero daqueles para quem a morte é iminente ${ }^{(18)}$. Grande parte dos hospitais recebe representantes religiosos no auxílio ao doente em sua terminalidade, sendo suas intervenções focadas em confortar o paciente e melhorar seu bem-estar no contexto da dor espiritual e condição crítica, estendendo suas ações a pacientes e familiares ${ }^{(15)}$. Todavia, em tempos de pandemia, sabese que existe dificuldade inclusive na prestação do cuidado espiritual(19).

Desse modo, cabe-nos refletir sobre a espiritualidade que nesse momento se impõe. A espiritualidade corresponde à essência do ser humano, atributo inato, que promove bem estar, saúde e estabilidade, dando um novo sentido à vida de cada pessoa. Difere da religiosidade, sendo essa o modo do indivíduo expressar sua espiritualidade por meio da adoção de valores, crenças e práticas de rituais que fornecem respostas às perguntas essenciais sobre a vida e a morte ${ }^{(20)}$.

Pensando nos princípios filosóficos dos cuidados paliativos, que adotam o conceito de dor total como cerne da assistência paliativista, precisamos buscar meios pelos quais seja possível cuidar da espiritualidade de cada paciente e de seus familiares em meio à pandemia. É necessário assumir essa dimensão como uma prioridade para as estratégias tera $\neg$ pêticas, pois reconhecidamente é fonte de bem-estar e qualidade de vida para pessoas que viven $\neg$ ciam o processo de morrer ${ }^{(21)}$.

No momento crítico da pandemia, a abordagem dos cuidados paliativos tornase essencial com o propósito de possibilitar meios pelos quais seja possível gerenciar as situações de morte iminente, de modo a reconhecer e respeitar o sagrado de cada paciente, a sua crença/fé que conforta sua dor espiritual. Esse é um dos pilares dos cuidados paliativos, mesmo reconhecendo as dificuldades e fragilidades devido à falta de tempo em meio à dinâmica dos hospitais comuns e mais especificamente dos de campanha, cenário desenvolvido para assistir os pacientes com Covid-19. 
Assim, devemos retomar a estética do cuidar, a sensibilidade de fazer o bom uso das tecnologias de modo a permitir que o paciente possa ter contato com seus familiares ou líderes espirituais por meio de suportes tecnológicos e, assim, cuidar da dimensão espiritual de cada paciente hospitalizado, mitigando os traumas decorrentes de uma morte distanciada e sem a permissão de despedidas ${ }^{(4)}$.

Discorrer sobre paliação na terminalidade da vida está associado ao ajuste de algoritmos e relacionamentos. Porém, em momentos oportunos, os algoritmos são colocados como primordiais na execução desses cuidados; a presença de um profissional psicólogo da paliação fornecerá contato com os familiares no pós-morte, visto que o contato humano não é permitido a pacientes em estado grave acometidos pela Covid-19. A prática de novos setores intra-hospitalares dedicados aos cuidados de fim de vida em meio à crise da Covid-19 tem possibilitado o gerenciamento compassivo, mediado pela tecnologia de forma adequada, proporcional e ética ${ }^{(4)}$.

Nesse contexto de sofrimento familiar resultante da dor emocional decorrente da perda, assim como dos doentes em estado iminente de morte distante dos seus entes queridos, a aflição é visível em ambos os lados. O profissional, além de prestar os cuidados, precisa trabalhar com familiares e pacientes a necessidade do isolamento, e, ao mesmo tempo, proporcionar a todos momentos de amor e compaixão, estimular a reflexão e proporcionar meios para momentos do cuidado à espiritualidade, na busca por amenizar o sofrimento causado pelo contexto situacional.

Com isso, a prática da espiritualidade em unidades intensivas reflete seus cuidados na qualidade da assistência prestada ao paciente/família, compreendendo tratar-se de um ambiente que, devido ao grau de tensão, carrega em seu estigma a construção cultural de medo do desfecho da doença com a provável proximidade da morte, podendo gerar na família uma crise emocional caracterizada por ansiedade e estresse relacionados com a ida do seu ente para a Unidade de Terapia Intensiva (UTI), como tem ocorrido em muitos momentos. Desse modo, compreendemos que uma assistência de qualidade, pautada em um relacionamento interpessoal satisfatório com pacientes e familiares, torna o ambiente mais humanizado e o cuidado focado em valorizar o ser humano como ser integral(22-23).

A atual pandemia remete ao fato de que, enquanto humanos, somos mutuamente dependentes uns dos outros. Ninguém pode se cuidar sozinho, sempre dependerá de outros, de muitos, de todos. Somos seres criados para a solidariedade, para a conexão e para o afeto. O tempo nos oportuna uma experiência de conectividade mais profunda com o mundo e com aqueles que realmente importam, e nos conduz para uma relação transcendental da alma com a divindade.

Sendo assim, no confrontamento de situações de dor e sofrimento, como nos cuidados de fim de vida, os relacionamentos interpessoais são ressignificados, seja com a família ou com a equipe de cuidados paliativos. A essência do cuidado está associada às proximidades e vínculos, os quais encontram apoio na espiritualidade, na transcendentalidade ${ }^{(24)}$.

\section{CONSIDERAÇÕES FINAIS}

Há uma lacuna nos estudos relacionados à espiritualidade e ao processo de morrer e morte, em especial no cenário da pandemia, apesar dos vários questionamentos a respeito dos sentimentos ocasionados com o isolamento e/ou distanciamento social, sofridos por parte dos doentes afastados dos familiares e vice-versa.

Avaliar de forma segura a dimensão espiritual das pessoas para suportar a dor da perda de seu familiar, podendo essa ser temporária ou definitiva, é fundamental. É inerente trabalhar no campo da espiritualidade, orientar, conversar ou diminuir a dor espiritual do sofrimento através de intervenções terapêuticas que respeitem as diversas crenças e os dogmas religiosos de cada ser humano. Saber que espiritualidade e religiosidade 
se diferem, mas que precisam ser levadas em consideração sempre, com o respeito ao sagrado de cada pessoa.

A equipe de saúde, em especial do campo da Enfermagem, pela maior proximidade com o paciente, pode e deve enfatizar o uso das intervenções terapêuticas com foco na espiritualidade, valorizando a presença plena de um ou mais membros da equipe de saúde, de preferência um profisșional que se identifique com uma conexão entre este e a espiritualidade do pacientes. É importante até mesmo o reconhecimento dos próprios limites da prática cotidiana, o que conduzirá para a necessidade de um acompanhamento especializado, que pode ser fornecido por um capelão ou um líder religioso/espiritual, à escolha do paciente. Essa intervenção pode ser materializada com o uso das mediações tecnológicas, possibilitando o encontro virtual entre paciente e o líder espiritual de sua escolha.

Pacientes vítimas de Covid-19 em estado avançado da doença, em cuidado de fim de vida, têm o mesmo direito de assistência e atenção comparados àqueles que estão saindo do quadro crítico por melhora da sintomatologia. Comparados a outros doentes, requerem adequado controle dos sintomas ou uma morte digna, princípio básico e constitucional de todo cidadão. Todo profissional de saúde, na qualidade de cuidador, tem o dever fundamental de aliviar o sofrimento, adequando atendimento favorável a partir dos recursos disponíveis, assim como atenção na espiritualidade, independentemente das chances de sobrevivência, reconhecendo que essa dimensão integradora jamais poderá ser esquecida, por ser parte da nossa essência como seres humanos.

\section{REFERÊNCIAS}

1. World Health Organization (WHO). Novel Coronavirus technical guidance 2020. Genebra: WHO; 2020. [Internet]. 2020 [acesso em 14 abr 2020]; Disponível em: https://www.who.int/emergencies/diseases/ novel-coronavirus-2019.

2. World Health Organization (WHO). The world health report 2002. Genebra: WHO; 2002.

3. Girardi G. O Estado de São Paulo. Estudo prevê ao menos 44 mil mortes de Covid-19 no Brasil. [Internet]. 2020 [acesso em 27 mar 2020]; Disponível em: https://saude.estadao.com.br/noticias/ geral,estudo-preve-ao-menos-44-mil-mortes-de-covid-19-no-brasil-isolar-so-idosos-eleva-n-para-529mil,70003251026.

4. Bertè R, Cassinelli D, Vignola V, Bonfanti S, Pagano S, Costa A, et al. Covid-19 the role of palliative care had to be adapted to manage this "ultra-emergency". The BMJ opinion. [Internet]. 2020. [acesso em 31 mar 2020]; Disponível em: https://blogs.bmj.com/bmj/2020/03/31/covid-19-the-role-of-palliative-carehad-to-be-adapted-to-manage-this-ultra-emergency/.

5. Newery S. NHS considers cancelling non-emergency surgery to free up resources for coronavirus response. The Telegraph. [Internet]. 2020. [acesso em 31 mar 2020]; Disponível em: https://www. telegraph.co.uk/global-health/science-and-disease/nhs-considers-cancelling-non-emergency-surgery-freeresources/.

6. Zhang, W. Manual de Prevenção e Controle da Covid-19 segundo o Doutor Wenhong Zhang. São Paulo: Polo Books; 2020.

7. Lloyd-Sherlock P, Ebrahim S, Geffen L, Mckee M. Bearing the brunt of covid-19: older people in low and middle income countries. BMJ. [Internet]. 2020 [acesso em 21 abr 2020]; 368. Disponível em: https:// doi.org/10.1136/bmj.m1052.

8. Nunes VM de A, Machado FC de A, Morais MM de, Costa L de A, Nascimento ICS do, Nobre TTX, et al. COVID-19 e o cuidado de idosos: recomendações para instituições de longa permanência. Natal: EDUFRN; 2020. [Internet]. 2020. [acesso em 22 abr 2020]; Disponível em: https://repositorio.ufrn.br/jspui/ 
9. Lauer SA, Grantz KH, Bi Q, Jones FK, Zheng Q, Meredith HR, et al. The incubation period of Coronavirus disease 2019 (COVID-19) from publicly reported confirmed cases: estimation and application. Ann Intern Med. [Internet]. 2020 [acesso em 12 abr 2020]; 172(9). Disponível em: https://doi.org/10.7326/ M20-0504.

10. Huang C, Wang Y, Li X, Ren L, Zhao J, Hu Y, et al. Clinical features of patients infected with 2019 novel coronavirus in Wuhan, China. The Lancet.[Internet]. 2020 [acesso em 12 abr 2020]; 395(10223). Disponível em: https://doi.org/10.1016/S0140-6736(20)30183-5.

11. World Health Organization (WHO). Coronavirus Disease 2019 (COVID-19): Situation report - 38. [Internet]. 2020 [acesso em 12 abr 2020]. Disponível em: https://www.who.int/docs/default-source/ coronaviruse/situation-reports/20200227-sitrep-38-covid-19.pdf?sfvrsn=2db7a09b 4 .

12. World Health Organization (WHO). Statement on the second meeting of International Health Regulations (2005) Emergency Committee on the new Coronavirus outbreak of novel coronavirus (2019nCoV). [Internet]. 2020. [acesso em 15 de abr 2020]. Disponível em: https://www.who.int/news-room/ detail/30-01-2020-statement-on-the-second-meeting-of-the-international-health-regulations-(2005)emergency-committee-regarding-the-outbreak-of-novel-coronavirus-(2019-ncov).

13. Silva RS da, Campos AER, Pereira A. Caring for the patient in the process of dying at the Intensive Care Unit. Rev Esc Enferm USP. [Internet] 2011 [acesso em 12 abr 2020]; 45(3). Disponível em: https://doi. org/10.1590/S0080-62342011000300027.

14. Kovács MJ. Educação para a morte. Psicol Ciênc Profissão. [Internet]. 2005 [acesso em 12 abr 2020]; 25(3). Disponível em: https://doi.org/10.1590/S1414-98932005000300012.

15. Peters L, Cant R, Payne S, O'Connor M, McDermott F, Hood K, et al. How death anxiety impacts nurses' caring for patients at the end of life: a review of literature. Open Nurs J. [Internet]. 2013 [acesso em 12 abr 2020]; 7. Disponível em: https://doi.org/10.2174/1874434601307010014.

16. Bifulco VA, lochida LC. A formação na graduação dos profissionais de saúde e a educação para o cuidado de pacientes fora de recursos terapêuticos de cura. Rev Bras Educ Med. [Internet]. 2009 [acesso em 12 abr 2020]; 33(1). Disponível em: https://doi.org/10.1590/S0100-55022009000100013.

17. Bajwah S, Wilcock A, Towers R, Costantini M, Bausewein C, Simon ST, et al. Managing the supportive care needs of those affected by COVID-19. Eur Respir J [Internet] 2020 [acesso em 12 abr 2020]; 55(2000815). Disponível em: https://doi.org/10.1183/13993003.00815-2020.

18. McClain CS, Rosenfeld B, Breitbart W. Effect of spiritual well-being on end-of-life despair in terminallyill cancer patients. The Lancet [Internet]. 2003 [acesso em 12 abr 2020]; 361(9369). Disponível em: https:// doi.org/10.1016/S0140-6736(03)13310-7.

19. Berning JN, Poor AD, Buckley SM, Patel KR, Lederer DJ, Goldstein NE, et al. A novel picture guide to improve spiritual care and reduce anxiety in mechanically ventilated adults in the intensive care unit. AnnalsATS. [Internet]. 2016 [acesso em 12 abr 2020]; 13(8). Disponível em: https://doi.org/10.1513/ annalsats.201512-831oc.

20. Cavalheiro CMF, Falcke D. Espiritualidade na formação acadêmica em psicologia no Rio Grande do Sul. Estud. psicol. Campinas. [Internet]. 2014 [acesso em 12 abr 2020]; 31(1). Disponível em: https://doi. org/10.1590/0103-166X2014000100004.

21. Wachholtz $A B$, Keefe FJ. What physicians should know about spirituality and chronic pain. South Med J. [Internet]. 2006 [acesso em 12 abr 2020]; 99(10). Disponível em: https://doi.org/10.1097/01. smj.0000242813.97953.1c.

22. Rodríguez LMB, Velandia MFA, Leiva ZOC. Percepción de los familiares de pacientes críticos hospitalizados respecto a la comunicación y apoyo emocional. Rev Cuid. [Internet]. 2016 [acesso em 12 abr 2020]; 7(2). Disponível em: http://dx.doi.org/10.15649/cuidarte.v7i2.330. 
23. Longuiniere ACFDL, Yarid SD, Silva ECS. Influência da religiosidade/espiritualidade do profissional de saúde no cuidado ao paciente crítico. Rev Cuid. [Internet]. 2018 [acesso em 12 abr 2020]; 9(1). Disponível em: http://dx.doi.org/10.15649/cuidarte.v9i1.413.

24. Araújo MMT de, Silva MJP da. Communication strategies used by health care professionals in providing palliative care to patients. Rev Esc Enferm usp. [Internet] 2012 [acesso em 12 abr 2020]; 46(3). Disponível em: https://doi.org/10.1590/S0080-62342012000300014.

Recebido: 07/05/2020

Finalizado: 26/05/2020

Editora associada: Luciana Puchalski Kalinke

Autor Correspondente:

Maria da Conceição Quirino dos Santos da Silva

Universidade Estadual do Sudoeste da Bahia

Av. José Moreira Sobrinho, s/n - 45205490 - Jequié, BA, Brasil

E-mail: conceiçãoquirino@gmail.com

Contribuição dos autores:

Contribuições substanciais para a concepção ou desenho do estudo; ou a aquisição, análise ou interpretação de dados do estudo - MCOSS

Elaboração e revisão crítica do conteúdo intelectual do estudo - RSS

Aprovação da versão final do estudo a ser publicado - ABAV, RNSOB

\section{(c) (1)}

Este obra está licenciado com uma Licença Creative Commons Atribuição 4.0 Internacional. 